\title{
Zwischen Modebegriff und Zukunftstrend
}



VON STEFAN SPIEKER

Stefan Spieker studierte Betriebswirtschaft an der Universität Hamburg. Seine Diplomarbeit schrieb er über den Strukturwandel bei gemeinnützigen Organisationen. Danach war er als Gründer und Geschäftsführer der OrgaCom $\mathrm{GmbH}$ und weiterer Gesellschaften im Bereich der Softwareentwicklung und als Dozent für Gründungsberatung tätig. Parallel engagierte er sich in der ehrenamtlichen Vorstandstätigkeit beim Arbeiter-SamariterBund und beim Paritätischen Wohlfahrtsverband in Hamburg. Im Jahre 2005 wurde er kaufmännischer Leiter der FRÖBEL Gruppe, ab 2007 Geschäftsführer des FRÖBEL e.V. sowie mehrerer Tochtergesellschaften. Das Unternehmen betreibt in mittlerweile acht Bundesländern über 125 Kindertageseinrichtungen und Horte sowie Beratungsstellen und Projekte der Kinder- und Jugendhilfe. www.froebel-gruppe.de

\author{
Die Vielzahl der Veröffentlichungen unter \\ Stichworten wie "Social Entrepreneur", \\ "Sozialunternehmer", "Soziale Innovation", \\ "social impact" und "social business" lassen \\ vermuten, dass im Bereich der Sozialwirtschaft eine \\ grundlegende Veränderung stattgefunden hat.
}

Wer die aktuellen Presseberichte und Magazinbeiträge liest, bekommt den Eindruck von einer Welt junger, hervorragend ausgebildeter und international geprägter Menschen, die skalierbare Geschäftsideen entwickeln und damit die Welt im großen Stil zum Besseren verändern.

Der Kreis von jungen Sozialunternehmen, der sich um die Hauptakteure Ashoka, die Schwab Foundation, die Beteiligungsgesellschaft BonVenture und andere gebildet hat, ist überschaubar, hat aber schon beeindruckende Unternehmen und Organisationen auf den Weg gebracht. Bekannte Beispiele sind »Wellcome« von Rose Volz-Schmidt, die »Eltern-AG « von Meinrad Armbruster und »Deutschland rundet auf « von Christian Vater.

Ein paar Impressionen verdeutlichen, wie nah und doch wie fern sich die beiden Welten sind - die klassische Wohlfahrtspflege und die "neuen « Sozialunternehmer. Handelt es sich hierbei wirklich um zwei Welten oder gibt es viel mehr Gemeinsamkeiten, als es zunächst scheint?

\section{Multi-Stakeholder-Konferenz 2013}

Die »Multi-Stakeholder-Konferenz zur Förderung und Verbreitung von sozialen Innovationen « findet im $\mathrm{Fe}$ bruar 2013 im Allianz-Forum in Berlin-Mitte statt. Sie wird ausgerichtet vom Bundesministerium für Familie, Senioren, Frauen und Jugend und soll die Akteure aus der Wohlfahrtspflege und die "neuen "Sozialunternehmer zu einem fachlichen Austausch anregen. Eingeladen wurden Verbandsvertreter aus den Spitzenverbänden von Caritas, Diakonie, Arbeiterwohlfahrt und dem Paritätischen, Mitgliedsorganisationen der Verbände, Vertreter von Ministerien und Stiftungen sind ebenso vertreten wie junge Sozialunternehmen, Wissenschaftler und Vertreter von Beteiligungsgesellschaften. Eine bunte Mischung von Menschen, die sich reiben, diskutieren und miteinander streiten, aber auch zu guten Ergebnissen kommen.

Wie weit die Positionen hier auseinander liegen wird deutlich, wenn ein Vertreter der Wohlfahrtspflege sich abgrenzt: »Alter Wein in neuen Schläuchen - wozu brauchen wir diese Modewelle: jedes Projekt, das von renommierten Stiftungen wie der Aktion Mensch bewilligt wird, beinhaltet eine soziale Innovation." Angemerkt wird auch, dass die wenigen, aber immer wieder in der Presse genannten Gründungen im Ashoka-Umfeld im Vergleich zu der sehr großen Anzahl an Organisationen und Einrichtungen der klassischen Wohlfahrtspflege deutlich überbewertet seien und auch unter deren Mitgliedern viele Perlen zu finden sind. Andererseits beeindrucken in Vorträgen und Foren die Vielfalt, Kreativität, Darstellungsformen und die eingeschlagenen neuen Wege vieler Projekte und Entwicklungen der »Social Entrepreneure«. 
Im Verlauf der Konferenz wird dann deutlich, dass die Verbindungen und Berührungen zwischen den »Neuen « und der Wohlfahrtspflege immer enger werden, die Kooperationsbeziehungen dichter und seitens Paritätischem, Caritas und Diakonie erste gemeinsame Projekte gestartet werden. Offensichtlich lohnt es sich, miteinander ins Gespräch zu kommen.

\section{"social impact lab"}

Das Inkubatorprojekt "social impact lab« ging im Juli 2013 in Berlin-Kreuzberg über die Bühne. Im »Lab « arbeiten 15 bis 20 Gründerinnen und Gründer parallel an der Gründung und dem Aufbau »ihres" Sozialunternehmens. Eine der bekanntesten Gründungen ist wahrscheinlich die »Auticon « von Dirk Müller-Remus, die ausschließlich Menschen mit Autismus als Consultants im IT-Bereich beschäftigt. Die Teilnahme am Programm des Labs wird von öffentlichen und privaten Förderern unterstützt; ein großer Förderer ist das Software-Unternehmen SAP.

Das Projekt ermöglicht den Gründern, kostenfrei für bis zu acht Monate die Räume und Ressourcen des Gründungszentrums zu nutzen, Beratungs- veranstaltungen aufgeteilt werden muss. Einige der vorgestellten Geschäftsideen fallen direkt in den Arbeitsbereich der klassischen Wohlfahrtspflege.

Die Bandbreite der Gründertypen und Gründungsideen ist weit gefächert. In der Regel handelt es sich um Teams, teilweise auch um Einzelgründer: vom internationalen Gründerteam, die eine IT-gestützte Zertifizierungsidee für Gastronomiebetriebe nach ethischen, allergenfreien oder nachhaltigen Aspekten vorstellen, über einen Sprachwissenschaftler, der in jahrelanger Forschungsarbeit ein besonderes Konzept zur Förderung von Schulkindern entwickelt hat, das er verbreiten möchte, bis hin zum Tüftlerteam, das einen Prototyp von Spikes für Rollstühle entwickelt hat.

Der Auswahlprozess für dieses Förderprogramm orientiert sich stark an den Prozessen der klassischen Gründerförderung: Jeder Gründer oder jedes Gründungsteam hat fünf Minuten für die Vorstellung seiner Geschäftsidee und des Geschäftsmodells. Anschließend besteht die Möglichkeit, weitere zehn Minuten kritische Fragen zur Gründungsidee mit allen Teilnehmern offen zu diskutieren. Eine Jury wählt danach aus - und es gibt zugleich parallel eine Bewertung durch das Plenum aller

\section{"In der Gemeinschaftsküche des Gründerzentrums wird intensiv über Markttrends, Finanzierung und Marketing gesprochen"}

angebote in Anspruch zu nehmen und vor allem intensives Feedback von Mitgründern und Coaches zu erhalten. Die Atmosphäre wirkt auf jeden Besucher hoch kreativ und anregend, und das vor allem ist es, was diese Form der Gründungsunterstützung ausmacht. Nicht umsonst ist der zweitwichtigste Raum neben dem Großraumbüro die große Gemeinschaftsküche, in der intensiv über Markttrends, Finanzierung und Marketing gesprochen wird.

Im Sommer 2013 sind beim »social impact lab « unerwartet viele Bewerbungen eingegangen. Von diesen werden immer noch 20 zugelassen, weshalb die Auswahl der Gründungskandidaten, der sogenannte Pitch, auf zwei Nachmittags-
Zuhörer, die erstaunlicherweise in aller Regel dem Votum der Jury entspricht. Durch dieses Verfahren werden Schwächen der Gründungsidee aufgedeckt: Ist die Idee wirklich neu, sind offensichtlich Hürden und zu erwartende Probleme bereits bedacht und berücksichtigt worden, ist eine Alleinstellung (in der Regel ist dies die Innovation) erkennbar und findet sich für die Idee ein Markt?

Durch die Teilnahme vieler ehemaliger und aktueller Gründerinnen und Gründer auch als Zuhörer ist sichergestellt, dass diese ihre frisch gemachten Erfahrungen in die Diskussion einbringen - zum Nutzen der "pitchenden « Gründer und zur Verbesserung ihres Geschäftsmodells.
Obwohl viele Gründer ausführliche Recherchen für Ihr Gründungsprojekt vorgenommen haben und viel Idealismus, Kreativität und Ideenreichtum mitbringen, fällt dem Beobachter auf, dass Verbände und Organisationen der Wohlfahrtspflege bei der Vorstellung der von den Gründungsteams aufgebauten und identifizierten Netzwerke überhaupt nicht vorkommen, also unbeachtet bleiben. Offensichtlich sind die Verbände und ihre Mitgliedsorganisationen für diese Gründungsteams kaum sichtbar oder versprechen ihnen zunächst keinen Nutzen. Vor diesem Hintergrund bleibt für die Verbände und ihre Mitglieder noch einiges zu tun, um dieses Nachwuchsklientel zu erreichen.

Dass die Teilnehmer an dem Pitch, gleich ob als Gründer oder als Zuschauer. ein enormes Potenzial mitbringen, ist offensichtlich. Offen ist jedoch, ob diese jungen Gründer für die klassischen Organisationen der Wohlfahrtspflege erreichbar sind - oder zumindest in Zukunft erreichbar sein werden. Die Kreativität und Expertise, die diese Sozialgründer mitbringen, würden die klassischen Wohlfahrtsverbände erheblich bereichern - und das Potenzial dieser Menschen ist bei weitem nicht ausgeschöpft: Wir können auf einen aktuellen Trend setzen, dass junge, gut ausgebildete Menschen vermehrt ihre Berufe und Jobs danach auswählen, ob ein interessantes Umfeld und insbesondere ein gesellschaftlicher oder sozialer Mehrwert geschaffen wird. Dieser Trend wird nicht zuletzt auch durch die Imagekrise wichtiger Branchen wie beispielsweise der Banken, verstärkt, und die Wohlfahrtsverbände und ihre Mitgliedsorganisationen tun gut daran, diesen Trend für sich aktiv zu nutzen und Angebote für Entrepreneure und Berufseinsteiger aktiv zu entwickeln.

Die Auswertung dieser Eindrücke veranlasst mich zu einigen Feststellungen und Thesen:

\section{Erste These: Die Wohlfahrtspflege wirkt altbackener als sie ist}

In der Presse liest man immer wieder Stichworte wie »Wohlfahrts-Kartell«, »Wohlfahrts-Industrie « und »Dinosaurier«, wenn Themenfelder der Sozialen Arbeit ökonomisch beleuchtet werden. Die Verbände selber tun leider zu wenig, um diesem wertenden Eindruck entgegenzuwirken. 


\section{"Der Bürokratismus hemmt alte und neue Anbieter sozialer Dienstleistungen"}

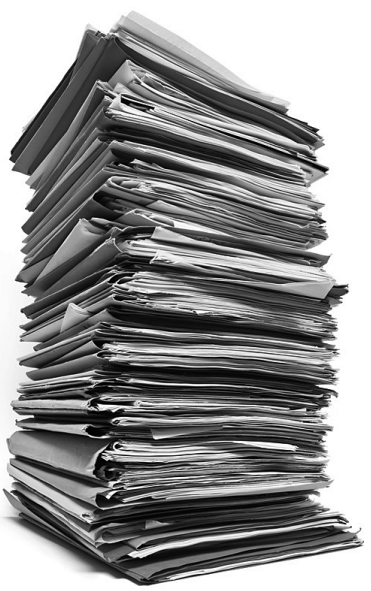

Viele Neugründungen in der Sozialwirtschaft stehen bald vor den gleichen bürokratischen Hürden wie die traditionellen Verbände und Träger sozialer Dienste und Einrichtungen. Einige Beispiele:

- Aufgrund von Hygieneauflagen für große Einrichtungen (HACCP) dürfen Kinder häufig nicht den zuhause gebackenen Geburtstagskuchen mit in die Einrichtung bringen, da kein Zertifikat vorhanden ist.

- Das Besserstellungsverbot verhindert, dass in öffentlich geförderten Einrichtungen der Wohlfahrtspflege besser gezahlt wird, als im öffentlichen Dienst. Dies ist insbesondere dann hinderlich, wenn bestimmte Qualifikationen und Leistungen besonders honoriert werden sollen. Leider hat sich mit dem TVöD auch die Besserbezahlung bei einfacher Seniorität ausgebreitet, wer also länger dabei ist, erhält mehr Geld - unabhängig von seiner Motivation, Leistung und Fortbildung.

- Jedes Förderprogramm ist mit einer Vielzahl von Regularien versehen, die sich gerne hinter Wort- und Buchstabenungetümen wie AnBestP, Landeshaushaltsordnung, Ausschreibungsverpflichtungen verbergen und die einen Umgang mit diesen Mitteln nur für erfahrene Verwaltungsleute möglich macht. Für innovative - also in der Regel kleine und junge - Organisationen ist die Anwendung dieser Programme sehr schwierig und setzt sicherlich eine weitgehende Anpassung an bürokratisches Denken und Handeln voraus. Ob diese Regelanpassung noch große Innovationskraft zulässt, ist zumindest zweifelhaft.

- Ausgeschriebene Förderprogramme erhalten feste Programmschemata und werden nur vergeben, sofern man sich an die - oftmals haarklein beschrie- benen - Ausschreibungsbedingungen hält. »Echte" Innovation, die sicherlich nicht durch allzu enge Programmbeschreibungen eingeschränkt werden darf, wird so nur bedingt möglich.

- Besonders innovationsfeindlich sind im Detail gegliederte Finanzierungsformen über die Spitzabrechnung. Vor lauter Angst, die Träger der Wohlfahrtspflege könnten auf die Idee kommen, Personaloder Sachkosten sachfremd zu verwenden oder gar in anderen Gewichtungen ausgeben, als in der aufsichtführenden Behörde vorgesehen wird die Zuwendungsfinanzierung eng gesteuert.

- Zu den gern angewandten Fußfesseln gehören auch Maximalgrenzen für Teilbudgets, die natürlich nicht oder nur bedingt passend fortgeschrieben werden. So entwickeln sich die Träger der Wohlfahrtspflege eher zu Optimierern von Finanzierungsbedingungen (wo, wie und wann muss/kann ich noch ein Teilbudget ausschöpfen und sichern) als zu Optimierern der täglichen Sozialen Arbeit. Schuld sind allerdings nicht die Verantwortlichen bei den Trägerorganisationen, sondern die oftmals widrigen und widersinnigen Rahmenbedingungen.

Stefan Spieker
Um die wirtschaftliche Bedeutung der Wohlfahrtspflege zu dokumentieren, wird gerne die Bedeutung von $\mathrm{Ca}$ ritas und Diakonie als größte deutsche Arbeitgeber dargestellt. Im Bewusstsein der Öffentlichkeit bleibt dann das oft und gern zitierte Bild des Sozialmolochs oder Wohlfahrtsdinosauriers hängen und vergessen wird die große Stärke der Verbände: Als Mitgliedsorganisationen und zivilgesellschaftliche Akteure binden sie eine Vielzahl von kleinen beeindruckenden Vereinen und Organisationen in ihre Arbeit ein, übernehmen ihre Interessenvertretung und unterstützen sie bei der laufenden Arbeit, und zwar schon bei Aufbau und Gründung.

Die Innovationskraft dieser Organisationen erreicht Medien und Öffentlichkeit viel zu selten, und spätestens seit dem unsäglichen Skandal um die Treberhilfe bekommt man leicht den Eindruck, dass Verbände und Organisationen in erster Linie unter Rechtfertigungsdruck stehen. Hier wäre es angezeigt, neben den immer wichtiger werdenden Transparenzberichten auch bewusst eine neue Form der Bericht- erstattung dagegenzustellen, beispielsweise einen Innovationsbericht, der aufzählt, in welchen Regionen oder Arbeitsfeldern die jeweilige Organisation erfolgreich wirkt. Schnell würde sich zeigen, welche innovativen Potenziale gerade auch bei den "kleinen " Mitgliedsorganisationen im Wohlfahrtsbereich vorhanden sind.

Potenziale für soziale Innovationen sind vielfältig vorhanden. Neben einer neuen Kombination der Leistungsfaktoren (andere Formen der Leistungserbringung) liegen Innovationspotenziale insbesondere in neuen Kombinationen von Angeboten: die Kombination von Angeboten für junge Menschen mit Angeboten für alte Menschen, die $\mathrm{Zu}$ sammenführung guter Ideen aus der (Sozial-) Wissenschaft mit Organisationen, die in der Praxis wurzeln. Das Zusammenbringen von Erfahrungen und Angeboten aus unterschiedlichen Kulturkreisen und natürlich auch die Implementation von IT-Technologien und Internet bei der Erbringung sozialer Dienstleistungen: Hier liegen überall neue Ideen, also: soziale Innovationen, quasi auf der Straße. Und die vielfältige und breit gefächerte Mitgliederstruktur der Verbände könnte als Innovationsmotor genutzt werden.

\section{Zweite These: Die Wohlfahrtspflege ist nicht innovationsfeindlich - die Rahmenbedingungen oftmals schon}

Die Wohlfahrtspflege hat in der Vergangenheit vielfältige Impulse gesetzt. Sie nimmt zivilgesellschaftliche Bewegungen und Entwicklungen auf und bietet Ihnen ein Umfeld und eine Lobby zur Umsetzung ihrer gesellschaftlichen und sozialen Ideen oder Vorhaben. Die Organisationen im Umfeld der Aidshilfe und viele Selbsthilfeorganisationen sollen hier als Beispiel genannt werden.

Jedes Jahr kommen viele neue Organisationen hinzu und beantragen als Vereine ihre Aufnahme bei Parität \& Co. Schnell jedoch müssen die Gründer feststellen, dass es eine Vielzahl von bürokratischen Hürden im Gemeinnützigkeitsrecht, im Zuwendungsrecht und im Vereinsrecht gibt, die es zu über- 
winden gilt. Dies betrifft natürlich auch die klassischen und vor allem auch die großen Träger, die immer wieder Gefahr laufen, sich allzu sehr von den öffentlichen Institutionen, mit denen sie aufgrund von Aufsichts- und Finanzierungsvorgaben ständig zu tun haben, mit latenter Bürokratitis infizieren zu lassen.

$\mathrm{Zu}$ den bürokratischen Fesseln gehört unter anderem eine Vielzahl von Auflagen, mit denen die tägliche soziale Arbeit reglementiert wird und die im Gegensatz zu den Erfahrungen aus der Praxis, oftmals am grünen Tisch der Gesetzgeber und Verwaltungsbeamten geboren werden, wie viele Beispiele zeigen können (vgl. Kasten »Der Bürokratismus hemmt alte und neue Anbieter sozialer Dienstleistungen«, S. 9).

\section{Dritte These: Die Wohlfahrtspflege braucht neue Zugänge für gut qualifiziertes Personal}

Leider ist festzustellen, dass in den Organisationen der Wohlfahrtspflege auch im Management - immer noch zu wenige Quereinsteiger zu finden sind, die aus anderen Branchen stammen, keine soziale Ausbildung oder nicht Sozialwissenschaften studiert haben.

Ein Grund dafür ist sicherlich in der schlechten Vergütung im sozialen Bereich zu sehen: Soziale Organisationen und Träger haben es schwer, mit Vergütungsstandards in der Industrie oder in der Finanzbranche zu konkurrieren. Auch die Tatsache, dass nur einige wenige Berufsfelder im sozialen Bereich über den Weg der dualen Ausbildung erreichbar sind, führt dazu, dass junge Leute eher eine (wenn auch gering) vergütete Lehre absolvieren, als sich für eine Fachschulausbildung ohne Vergütung oder Förderung zu entscheiden.

Fazit: Die Sozialbranche braucht dringend und vermehrt junge talentierte Nachwuchskräfte und vor allem Neuund Quereinsteiger, die den berühmten frischen Wind mitbringen: Wir brauchen Angebote für Banker und Anwälte, die gesellschaftlichen Mehrwert in sozialen Organisationen schaffen wollen, internationale Medien- und Marketingleute, die etwas für Nachhaltigkeit und soziale Entwicklung in Bewegung bringen möchten, Handwerker und Facharbeiter, die erkennen, dass sie lieber mit Menschen als mit Maschinen arbeiten wollen.

\section{Vierte These: Die Wohlfahrtspflege braucht eine eigenständige und aktive "Gründungsförderung"}

Die Industrie- und Handelskammern machen es, viele Großunternehmen in der Wirtschaft im Übrigen auch: Sie bieten über den Beratungsprozess hinaus aktive Gründungsunterstützung an und sorgen dafür, dass innovative junge Unternehmen neu an den Start kommen. Die Industrie- und Handelskammern haben dafür in den vergangenen Jahren sogenannte »One-Stop-Shops « geschaffen: Hier erhalten Gründer (und solche die es werden wollen) Beratung, Informationen, Coaching und Unterstützung aus einer Hand. Die Unterstützung von Arbeitsagenturen (Gründungsgeld), der Kreditanstalt für Wiederaufbau und Förderungen der Europäischen Union, beispielsweise Beratungsgutscheine, werden gebündelt. In einigen Städten existieren sogenannte »Gründungsinkubatoren ", in denen Gründer in der ersten Phase ihrer Gründung zusammenarbeiten können, sich austauschen, Beratungsangebote erhalten, Seminare wahrnehmen können und in erfahrenen Unternehmern Mentoren und BusinessAngels finden. Warum können wir Vergleichbares nicht auch in der Sozialwirtschaft organisieren?

\section{Meine Vision}

Mein Vorschlag: eine »Sozialgarage «, in der unsere Verbände junge und talentierte Gründer fördern, gemeinwesenorientierte und gemeinnützige Geschäftsmodelle auf den Weg zu bringen.

Damit wären wir wieder beim »social impact lab « in Berlin-Kreuzberg. Dieser Inkubator macht im Grunde nichts anderes, als bekannte Gründungsförderer auch - nur bezogen auf Gründungen im Gemeinnützigkeitsbereich oder Gründungen mit einem Nachhaltigkeitsgedanken und einem besonderen gesellschaftlichen Mehrwert. Die besondere Leistung der Gründer dieses Zentrums besteht darin, das Thema des Sozialunternehmertums verbreitet zu haben und in der Gründerszene für die Sensibilisierung im Sinne des Sozialunternehmertums gesorgt zu haben.

Die Wohlfahrtsverbände könnten so etwas - gemeinsam mit ihren Mitgliedsorganisationen - ebenfalls einrichten, zumal sie viele dieser genannten Res- sourcen schon mitbringen: Erfahrene Sozialunternehmer können als Mentoren wirken, Gründungsberatung leisten Verbände in der Regel schon, und Coachingressourcen können eingekauft werden. Und nicht zuletzt mit einer Vielzahl von gelungenen und ausgezeichneten Aktionen, wie kürzlich der für »Mehr Männer in Kitas", haben die Verbände und ihre Mitgliedsorganisationen bewiesen, dass sie erfolgreich Kampagnen durchführen können, um neue Bevölkerungsgruppen für ihre Arbeit zu interessieren.

Die Wohlfahrtsverbände sollten sich trauen, gemeinsam solche Inkubatorprojekte zu starten - in jedem Falle wäre es einen Versuch wert ...

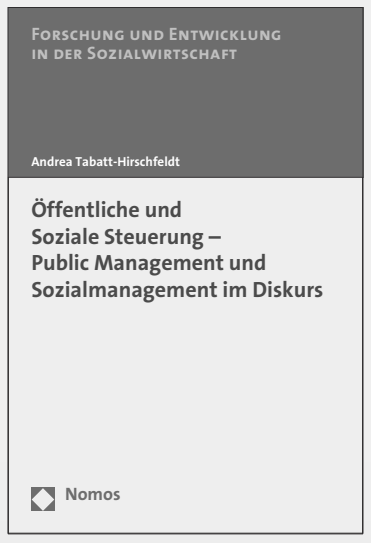

\section{Öffentliche und Soziale Steuerung - Public Management und Sozialmanagement im Diskurs}

Herausgegeben von Andrea Tabatt-Hirschfeldt

2013, ca. 186 S., brosch., ca. 34, - € ISBN 978-3-8487-0155-1

(Forschung und Entwicklung in der Sozialwirtschaft, Bd. 8)

Erscheint ca. Dezember 2013

www.nomos-shop.de/20302

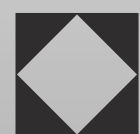
Nomos 